\title{
Wideband Environmental Electromagnetic Wave Observation Searching for Seismo-electromagnetic Signals and Simultaneous Observation of Catfish Behavior -The Cases for the Western Tottori and the Geiyo Earthquakes-
}

\author{
Chihiro Yamanaka, Hiroshi Asahara, Hiroshi Matsumoto and Motoji Ikeya
}

Department of Earth and Space Science, Graduate School of Science, Osaka University

Machikaneyama 1-1, Toyonaka, Osaka, 560-0043, Japan

\begin{abstract}
A detection system for environmental pulsed electromagnetic (EM) waves and some observation results were described. The system was a combination of wide band field meters capable to detect the absolute field intensity for three directions and electromyogram sensors for aquatic animals to monitor their activity continuously. Unusual coincidence between EM pulses and violent movements of a catfish was observed at Osaka 8 days before the Western Tottori Earthquake (Oct. 6, 2000, $M=7.3$ ). Unusually strong EM pulses were also detected at Shimane one day before the Geiyo Earthquake (Mar. 24, 2001, $M=6.7$ ). Evaluations of such pulse fields before earthquakes were made based on the hypothesis of pulsed charge generation due to preseismic stress changes. Possible physical relationship between the EM pulses and the Geiyo Earthquake was discussed based on multi dipole emissions of EM pulses in the stress changing zones.
\end{abstract}

Keywords: seismo-electromagnetic signals (SEMS), environmental electromagnetic wave, three direction sensor, catfish activity

\section{Introduction}

Precursory anomalies before earthquakes (EQs) have repeatedly interested people after big EQs. They have been studied scientifically, especially since mid 1970's after the success of forecasting of the Haicheng EQ in China (Feb. 4, 1975, $M=7.3$ ). After the Kobe EQ (Jan. 17, 1995, $M=7.3$ ), similar discussion has been actively continued among researchers (Hayakawa and Fujinawa, 1994; Ikeya, Furuta et al., 1996; Ikeya, Matsuda et al., 1998; Hayakawa et al., 1996; Hayakawa, 1999).

Previously, several hypotheses were offered to explain these precursors. Tributsch's "charged aerosols" hypothesis (Tributsch, 1978) are well known but the short lifetimes and slow diffusion rates of charged-aerosols may be hard to explain the widely affected area by precursor anomalies. Ionization by radon gas may cause similar anomalies, but the lifetime of radon about 3 days should have affected the duration of anomalies to be much longer.

One of the convincing physical mechanisms to explain the EQ precursors is electromagnetic (EM) effect considering the previous reports on precursor anomalies of EQs. (Ikeya and Matsumoto, 1997). Such electromagnetic phenomena relating to EQs are called seismo-electromagnetic signals (SEMS). In the case of the Kobe EQ, malfunctions of electric appliances and noises on TV before the EQ were reported as well as abnormal animal behavior. The former fact suggests that electromagnetic interferences (EMI) due to SEMS had occurred at the time (Matsumoto et al., 1998; Ikeya and Matsumoto, 1998). Abnormal animal behavior prior to the EQ is also explained as EM stimulation; certain aquatic animals have exquisite electrical 


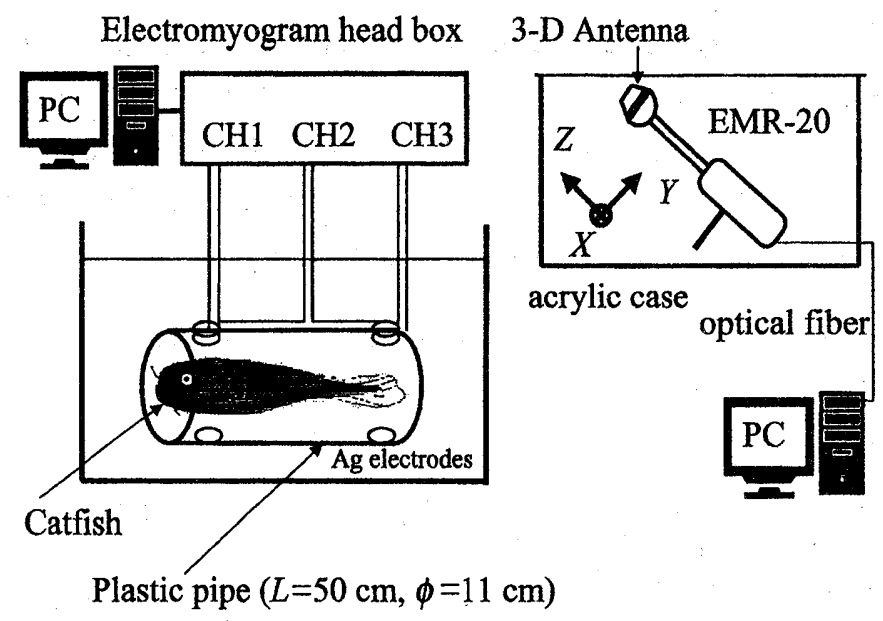

Fig. 1: A model of observation system. Activity of an aquatic creature is monitored by electromyogram (left) and environmental electric fields are monitored by 3-dimensional field sensors (right). EMR-20 detects frequency from $100 \mathrm{kHz}$ to $3 \mathrm{GHz}$, while EFA-2 covers the range from $5 \mathrm{~Hz}$ to $30 \mathrm{kHz}$.

sensitivity, and even terrestrial animals show abnormal behavior under electrical pulses (Ikeya, Furuta et al., 1996; Ikeya, Takaki et al., 1996; Ikeya, Matsuda et al., 1998).

However, the current scientific consensus on the "earthquake precursors" is quite reserved and most of the phenomena appearing in folklore and anecdotes are not categorized as earthquake precursors. (Wyss and Booth, 1997). Unfortunately, such precursor phenomena were not recorded at every earthquake and EQ predictions using such uncertain precursors have been considered not reliable. Apart from the practical EQ prediction, we would like to discuss the scientific explanation on unsolved EQ precursors and possible relations between SEMS and abnormal animal behavior: they are so often reported, sometimes recorded even before EQs. Therefore, it is not reasonable that all of them are just accidental happenings or retrospective illusions.

A number of efforts have been made to observe SEMS from DC to VHF bands. Most of the researches are based on narrow band measurements to eliminate EM waves from other sources. Different researchers use different methods at various wavelengths. ULF or ELF emissions before EQs have been observed by many researchers. For example, the perpendicular component of magnetic field more than $50 \mathrm{nT} / \sqrt{ } \mathrm{Hz}$ at frequency range of $0.01 \mathrm{~Hz}$ was observed before the Loma-Prieta EQ (Fraser-Smith et al., 1990). Another interesting result obtained at VLF bands pointed out that preseismic EM emissions were distinguished from those from lightning, because preseismic emissions were originated from a fixed position around the epicenter of the imminent EQ (Asada et al., 2001). Observation of scattered FMradio waves is also being studied in Japan (Kushida and Kushida, 1998).

Physical bases of SEMS are now still at stages of hypotheses. One hypothesis is based on the charge generation due to the stress-changes before large slip displacement. Our theory of released piezo-compensating charges was presented earlier (Ikeya, Takaki et al., 1997). If we assume abrupt changes of stress in certain area, the emissions of EM waves will have pulselike shapes containing wider frequency range. Such EM emission should be observed at multi frequencies or at wide frequency. The changes of magnetic field observed before the LomaPrieta EQ would have been stronger if it were observed at wider frequency. 


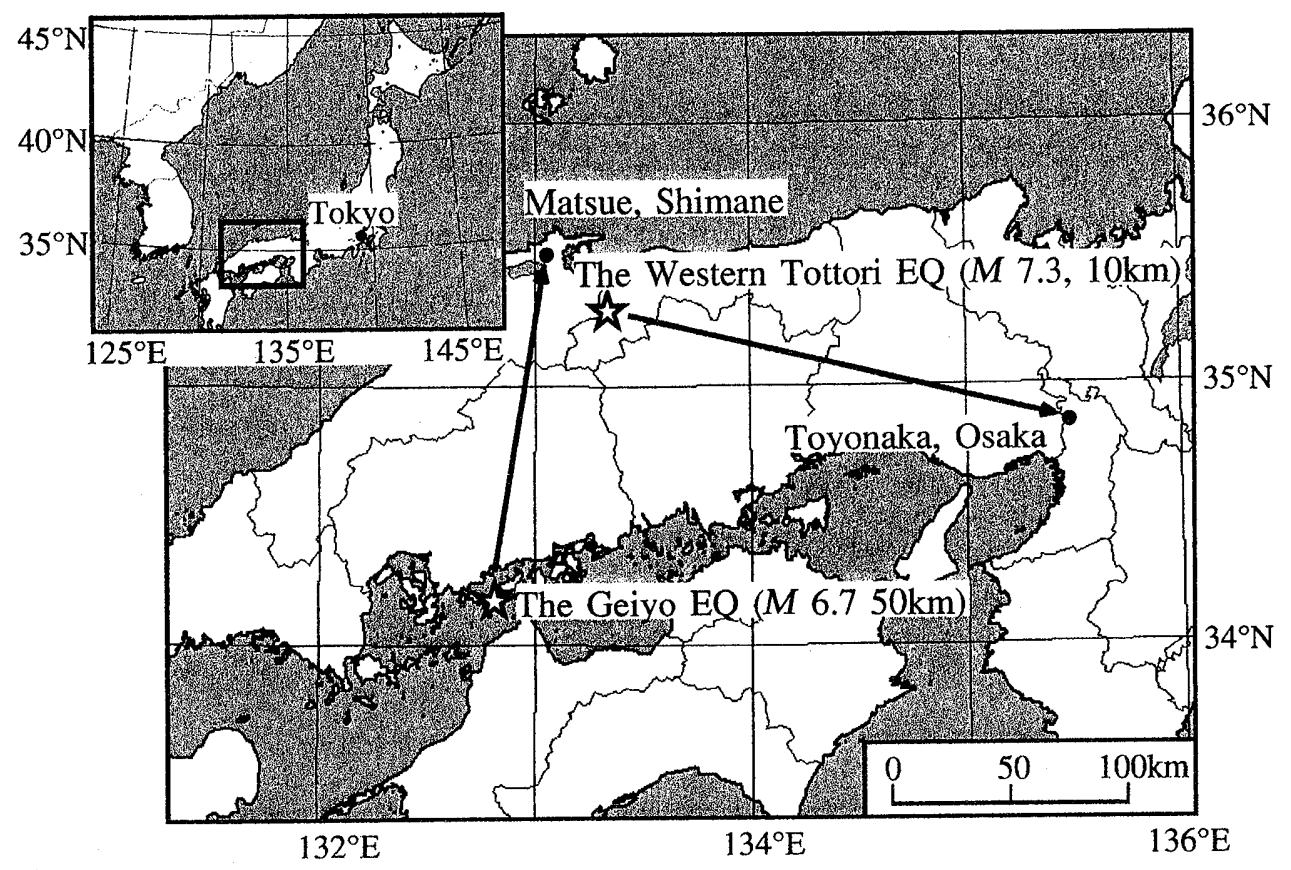

Fig.2: Map indicating monitoring sites. Low frequency measurements with EFA-2 are performed at Osaka only. Stars indicate the recent earthquakes studied in this research.

Here, we report about our recent system to record absolute values of environmental EM field in three directions (3-D) and at wide frequencies. Also, we will discuss the data on two recent EQs in western Japan: the Western Tottori EQ $(M=7.3$, Oct. 6, 2000) and the Geiyo EQ $(M=6.7$, March 24, 2001).

\section{The measuring system}

\subsection{EM sensors}

Figure 1 shows an example of observation system. Commercial three-dimensional field meters (Wandel \& Goltermann, Germany) are used to detect environmental EM waves. Each EM field meter is placed in a fixed acrylic box to isolate from human access and to prevent artifacts or near-by static electric discharging.

EMR-20 and EMR-200 with type-8 electric field probes (Wandel \& Goltermann) are capable to detect the 3-D electric fields of $0.6-800 \mathrm{~V} / \mathrm{m}$ from $100 \mathrm{kHz}$ to $3 \mathrm{GHz}$ using 3 dipole sensors with diodes. Information of the instantaneous electric fields on 3-D is sampled with 400 $\mathrm{ms}$ intervals and is transferred to a local computer with optical serial cables. Obtained data are stored in a hard disk with the time codes at every two second. All the clocks of computers for the measurement are adjusted to an atomic clock via the world wide web. Data stored in a local PC are automatically sent to the main PC for processing at every 6 hours through the Internet.

EFA-2 with a type-B field probe (Wandel \& Goltermann) is used for magnetic field measurements at the levels from $10 \mathrm{nT}$ to $10 \mathrm{mT}$ and frequency from $5 \mathrm{~Hz}$ to $32 \mathrm{kHz}$ by means of three-dimensionally oriented coils and a digital signal processor (DSP). The device measures effective magnetic field with a notch filter at $60 \mathrm{~Hz}$. The data are transferred to a different PC 
at every two second. Originally, the EM sensors were designed to be used with batteries, but we added a stable DC power supply and a shielded power line for long-term continuous measurements. Operation with the DC-power supply was compared with that of another batteryoperated device at the same location and we have confirmed little difference in the performance between these two devices.

Continuous measurements with EM sensors were started at Osaka University (Toyonaka, Osaka Prefecture, N $34^{\circ} 46^{\prime}$, E $135^{\circ} 28^{\prime}$ ) from April 2000 after checking the equality of sensitivity using two sets of sensors. Another site at Shimane University, (Matsue, Shimane Prefecture, N 35 $27^{\prime}$, E $133^{\circ} 04^{\prime}$ ) has been operated since March 2001. This site was selected by considering the seismic gaps in western Japan where EQs following the Western Tottori EQ were expected. The EFA-2 for low frequency measurements has been performed only at Osaka since October 20, 2000. These sites are indicated in Fig. 2.

\subsection{Electromyogram for catfish}

Response of catfish to the environmental EM field is also monitored by a sensitive potentiometer for EMG (electromyogram) and a voltage amplifier 5103 (NF Circuits Corporation, Japan). The EMG is used to monitor the activity of a catfish that stays mainly in the nest made of a plastic pipe at the bottom of an aquarium as shown in Fig.1. The EMG can convert the movement of catfish to electric voltages up to $5.12 \mathrm{mV}$ by picking up potential changes with 3 to 7 pairs of $\mathrm{Ag}$ electrodes on the pipe. Data of activity are obtained with the sampling frequency of $25 \mathrm{~Hz}$, then transferred to a PC and recorded continuously in a hard disk with time codes. Another aquarium filled with only water is also monitored as a control. Continuous measurements by electromyogram started in Apr. 1, 2000 at Osaka University and in Nov. 1, 2000 at Shimane University.

Most natural electromagnetic waves are attributed to thunder lightning with the peak frequency around $10 \mathrm{kHz}$ due to the typical discharge lengths (MacGorman and Rust, 1998). The range of our EM detection system includes these frequencies, though no record that exceeds 1 $\mathrm{V} / \mathrm{m}$ due to lightning has been observed since the start of the measurement at Osaka. Lightning detection information by power companies is often referred for eliminating the noise.

\section{Results}

\subsection{Daily Performance}

\section{EM sensors}

Originally, the EM sensors are designed to monitor continuous EM waves. Therefore, the sensitivity of EM sensors to short pulses was investigated using a delayed pulse generator, an antenna and a radio frequency amplifier. Electric fields obtained for various length of pulse trains were compared with those obtained for continuous waves at $0.1-10 \mathrm{MHz}$. A result for EMR-20 is shown in Fig. 3. The system showed reasonable data for the pulse width more than 0.4 seconds. Short pulses less than 0.4 second caused apparently low field intensity. Therefore, the obtained field values in such cases would mean the lower-limit of real field intensity.

Operating background was usually $0.3-0.4 \mathrm{~V} / \mathrm{m}$ at open space in Osaka. This is beyond the detection levels of the instrument specification, though the actual intrinsic noise level in a shield box indicated to be $0.2-0.3 \mathrm{~V} / \mathrm{m}$. The obtained background was quite reasonable considering the local EM fields of broadcastings and microwave communications. A cell-phone near the EMR sensor (EMR-20, $<1 \mathrm{~m}$ ) gave the field of $1-3 \mathrm{~V} / \mathrm{m}$.

Figure 4 shows an example of background data for 24 hours at Osaka and Shimane, respectively. Unknown spikes $0.8-2.5 \mathrm{~V} / \mathrm{m}$ appeared a few times a day at Osaka, while the 


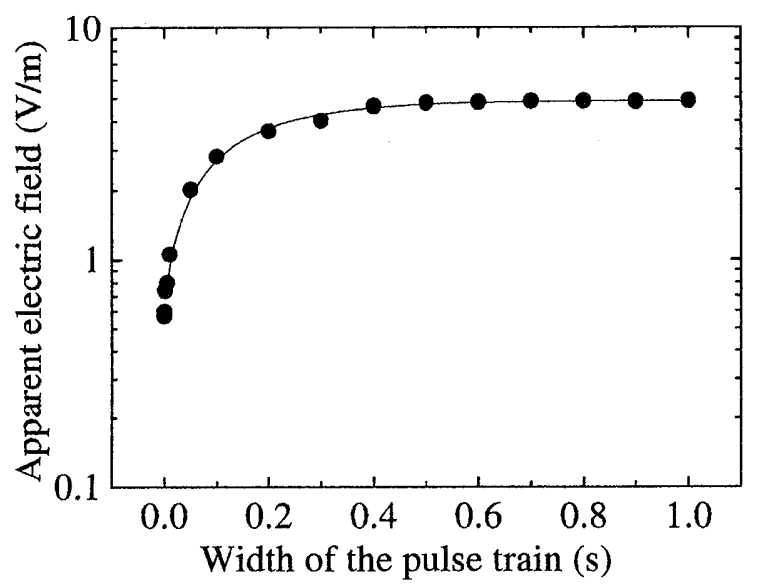

Fig.3: Response of electric field sensor EMR-20 to short pulses. External electromagnetic pulses were given using a radiowave antenna and an amplifier with gated sinusoidal electric waves. The frequency of the carrier sinusoidal wave was set at $1 \mathrm{MHz}$. Various lengths of pulse trains were applied to EMR-20 by changing the width of gated pulses to the amplifier. EMR-20 indicated apparently lower fields for shorter pulse trains less than 0.4 seconds.
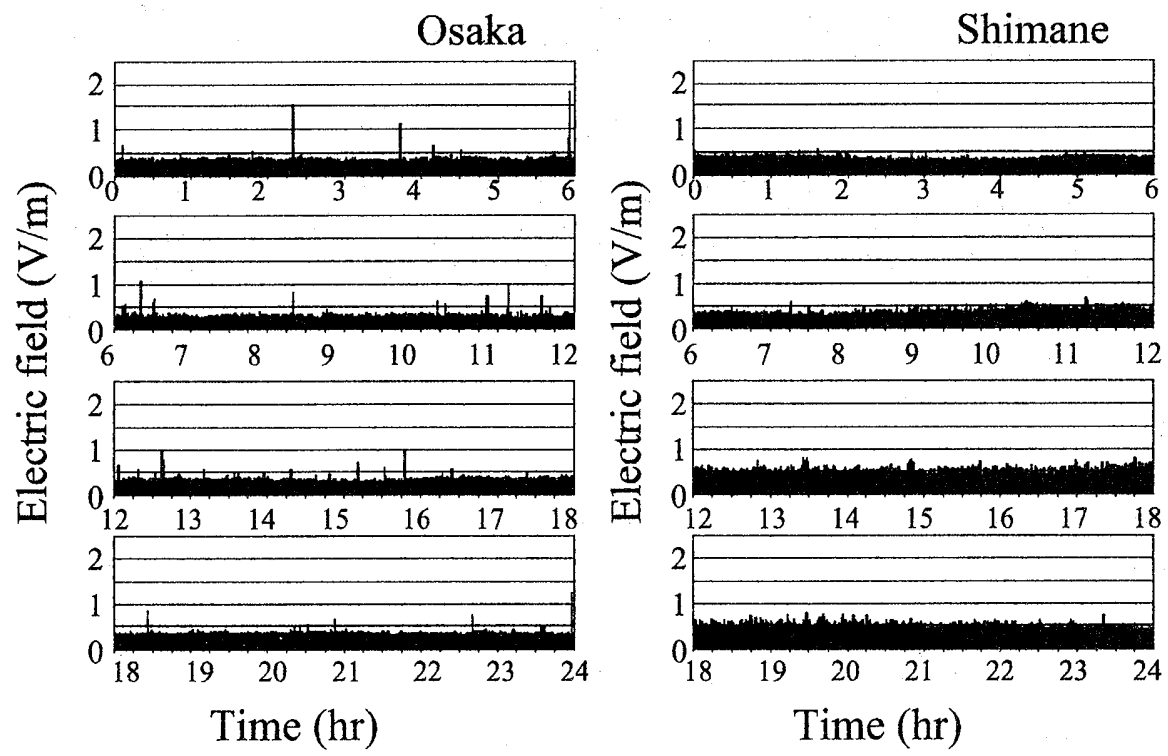

Fig.4: The electric field for one day monitored by EMR-20 at Osaka (left) and by EMR-200 at Shimane (right) obtained on April 17, 2001. Time resolution was 2 seconds each. Note spikes more than $1 \mathrm{~V} / \mathrm{m}$ were frequent at Osaka.

data at Shimane were rather stable. Such spikes appear depending on the day. They are probably mainly due to human activity like illegally strong mobile ham or near-by static discharges.

Results for 1 month as 10 minute averages are shown in Fig. 5. The Data at high frequency indicate daily rhythms of background fields, which is obvious at Shimane. As shown in Fig. 5, data of EFA-2 (4-30kHz magnetic field) at Osaka are quite stable with a background of 0.04 
$\mu \mathrm{T}$. Very few fluctuations on the data of EFA-2 mean that the low frequency noise from commercial power lines did not affect the measurements.

Catfish monitoring by electromyogram (EMG) and a numeration algorism

The background level of EMG measurements was about $10 \mu \mathrm{V}$ for the aquarium only filled with water. The EMG showed the level of $50 \pm 10 \mu \mathrm{V}$ for a slight movement of gills of the catfish. We have set the criteria of anomalies for violent movements as follows.

At each sampling $(25 \mathrm{~Hz})$ point, the obtained potential value is varying from less than 1 $\mathrm{mV}$ to more than $5 \mathrm{mV}$, so they are categorized in 6 steps with each step of $1 \mathrm{mV}$. We set the "instantaneous activity" of catfish at the sampling time to be integers from 0 to 5 , which are

4/11-5/10 Osaka
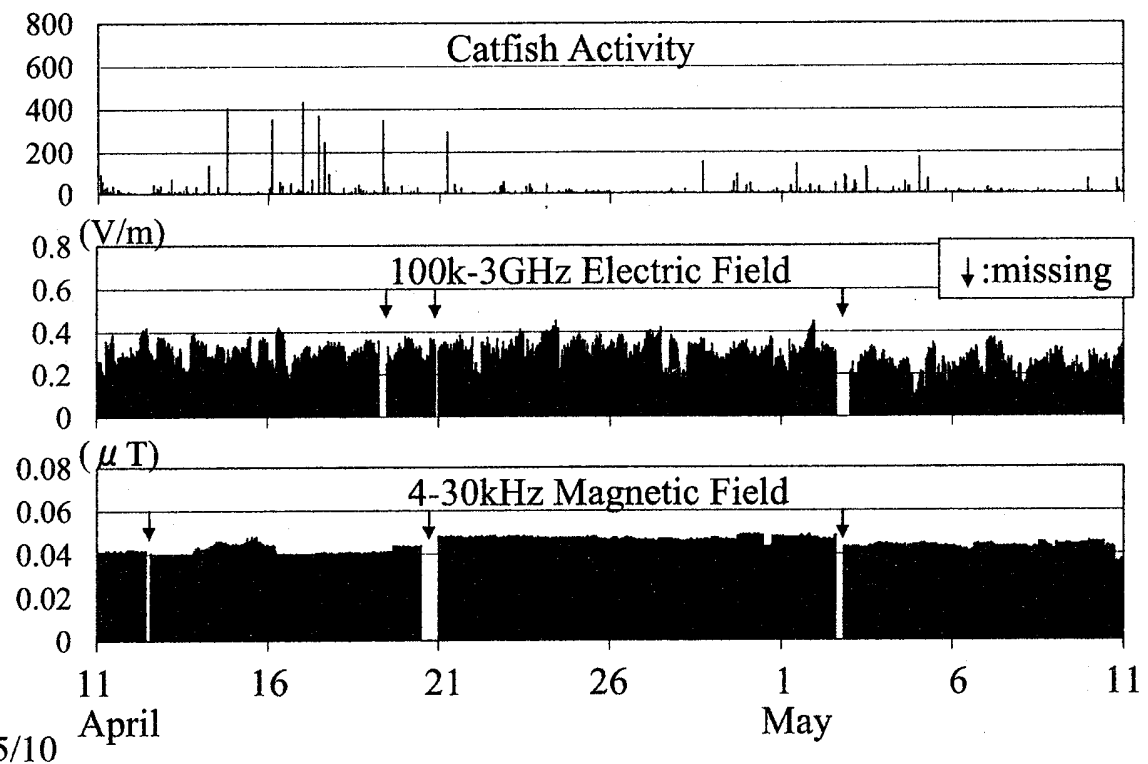

$4 / 11-5 / 10$

Shimane

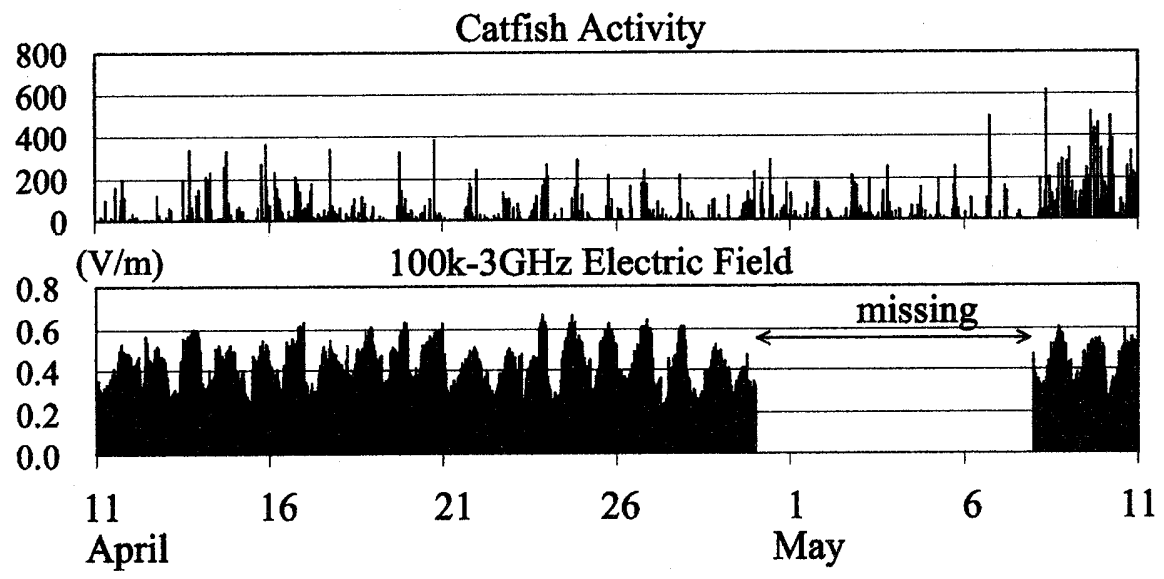

Fig.5: Observation of electromagnetic fields and catfish activity for one month (April 11 - May 10, 2001) at Osaka (top 3) and Shimane (bottom 2). Data are 10 minute averages. 
(a) Osaka

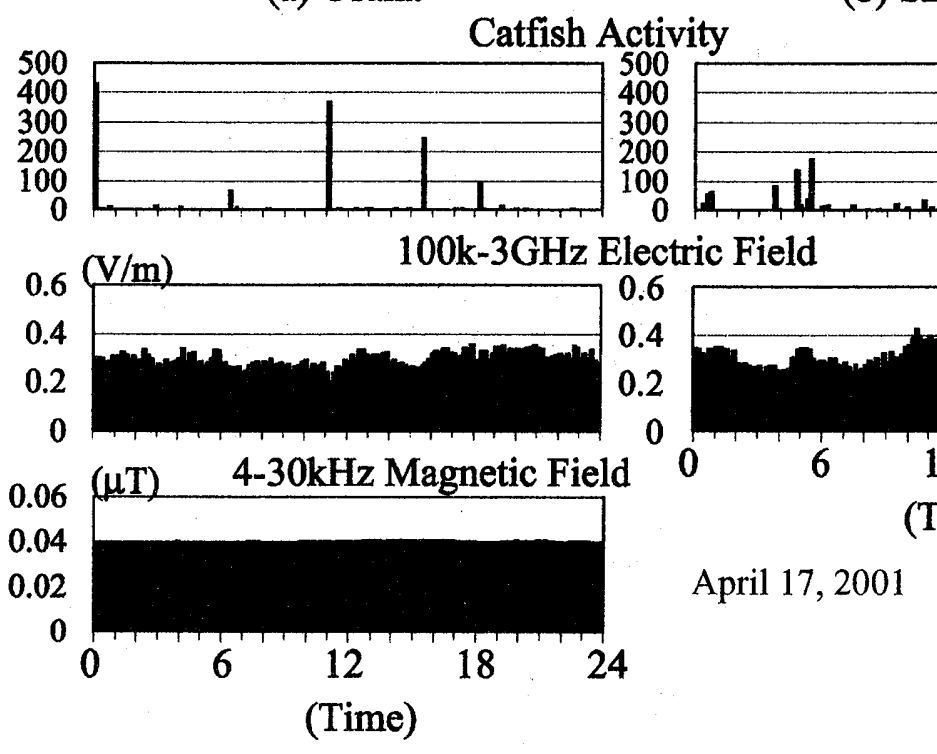

Fig.6: Electric fields and catfish activity for one day at Osaka (left) and Shimane (right) obtained on April 17, 2001. Plotted bars were 10 minute averages. Daily variation is obvious for high frequency electric field data at Shimane.

corresponding to the each 6 steps, respectively. The activity of catfish is counted by time integration of instantaneous activity for a series of movement. Following this method, usual activities such as gills movements are always indicated as zero. A movement such as moving out from the nesting pipe for feeding takes several seconds and is counted to be about 100 . A violent movement about 10 seconds causing spilling of water, for example, is counted as more than 1000. Conventionally, EMG data were monitored as 10 minutes average on a computer screen. A sudden violent movement of catfish for several seconds caused a clear change even in the averaged data. Then, the data in the range of interest are precisely examined to see the time-coincidence between the catfish activity and EM waves. Catfish activities of 10 minutes averages for one day are shown in Fig. 6 at both Osaka and Shimane.

\subsection{Observed results before earthquakes}

EM pulses and catfish activity before the Western Tottori Earthquake

Figure 7 shows the data obtained on Sep. 28, 2000 at Osaka, 8 days before the Western Tottori EQ (Oct. 6, 2000, $M=7.3$ ). The distance from epicenter to the detection site was about $190 \mathrm{~km}$. Although small spikes are appearing at times, the violent activity of the catfish and the high EM pulses coincided within one or two second at 3:57:54-55 $(1.13 \mathrm{~V} / \mathrm{m})$, and 5:08:55-57 $(1.22 \mathrm{~V} / \mathrm{m})$ of JST.

EM pulses before the Geiyo Earthquake

Figure 8 shows the data at both Osaka and Shimane on March 23, 2001, the one day before the Geiyo EQ (March 24, 2001, $M=6.7$ ). Clear spikes around $3 \mathrm{~V} / \mathrm{m}$ appeared at Shimane at 17:07, 17:20 and 17:22-23, while no change was observed at Osaka. The background level at Shimane was flat. The distance from epicenter to the Shimane site was $150 \mathrm{~km}$ and to Osaka was $350 \mathrm{~km}$, respectively. 
Catfish and EM pulse September 28, 2000.

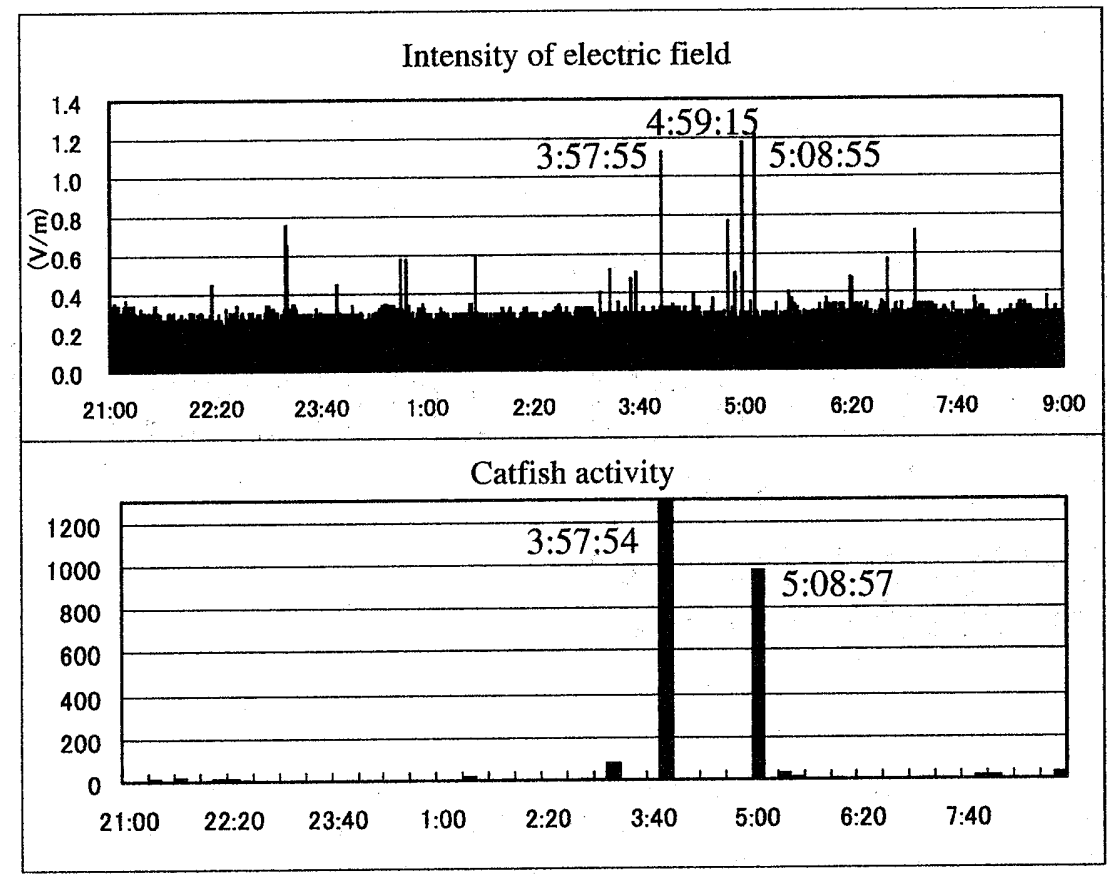

Fig.7: Observations at Osaka on Sep. 28, 2000, 8 days before the Western Tottori Earthquake (Oct. 6, 2000, 13:30 JST). Apparent coincidence of the pulse field at high frequency (top) and violent activities by catfish (bottom) were found at 3:57:54-55 and 5:08:55-57. The intensities of the electric field were above the background level but within the levels of usual spike noises. The Shimane site was not operating at this occasion.

\section{Discussion}

\subsection{Coincidence of EM pulse and Catfish activity}

As traditional Japanese legends remarked about the abnormal behavior of catfish before EQs, nocturnal Japanese catfish (silurus asotus) are quite sensitive to electric voltage of about $50 \mu \mathrm{V} / \mathrm{m}$ at $10-100 \mathrm{~Hz}$. The ability is used for finding prey. Asano (1998) studied the electric sensor of Japanese catfish and showed the minimum sensitivity of $5 \mu \mathrm{V}$. Such high sensitiveness, reaching almost the quantum limit, might be practically controlled by a complex parallel information processing system using other sensors to eliminate noises. Although statistical treatments are important in the study of animal behavior, we are concerned if we had "coincidence" phenomena between the violent behavior of the catfish and EM waves before EQs.

Coincidence phenomena of EM pulses and catfish activity were observed before the Western Tottori EQ as shown in Fig. 7. The probability of coincidence by chance was calculated assuming the phenomena of EM pulses and catfish movements were independent. Usually EM waves exceeding $1 \mathrm{~V} / \mathrm{m}$ were observed 1 to 40 pulses in a day at Osaka. Taking a largest value and considering the recording window of $2 \mathrm{~s}$, the probability of EM pulses in a day $\left(P_{1}\right)$ would be 80 seconds / 86400 second: $P_{1}=1 / 1080$. The activity of catfish, which exceeds 500 in terms of the activity defined above, happened once in ten days and each event typically lasts about 30 seconds: the probability $\left(P_{2}\right)$ would be $P_{2}=30 / 864000=3.47 \times 10^{-5}$. Therefore, the 

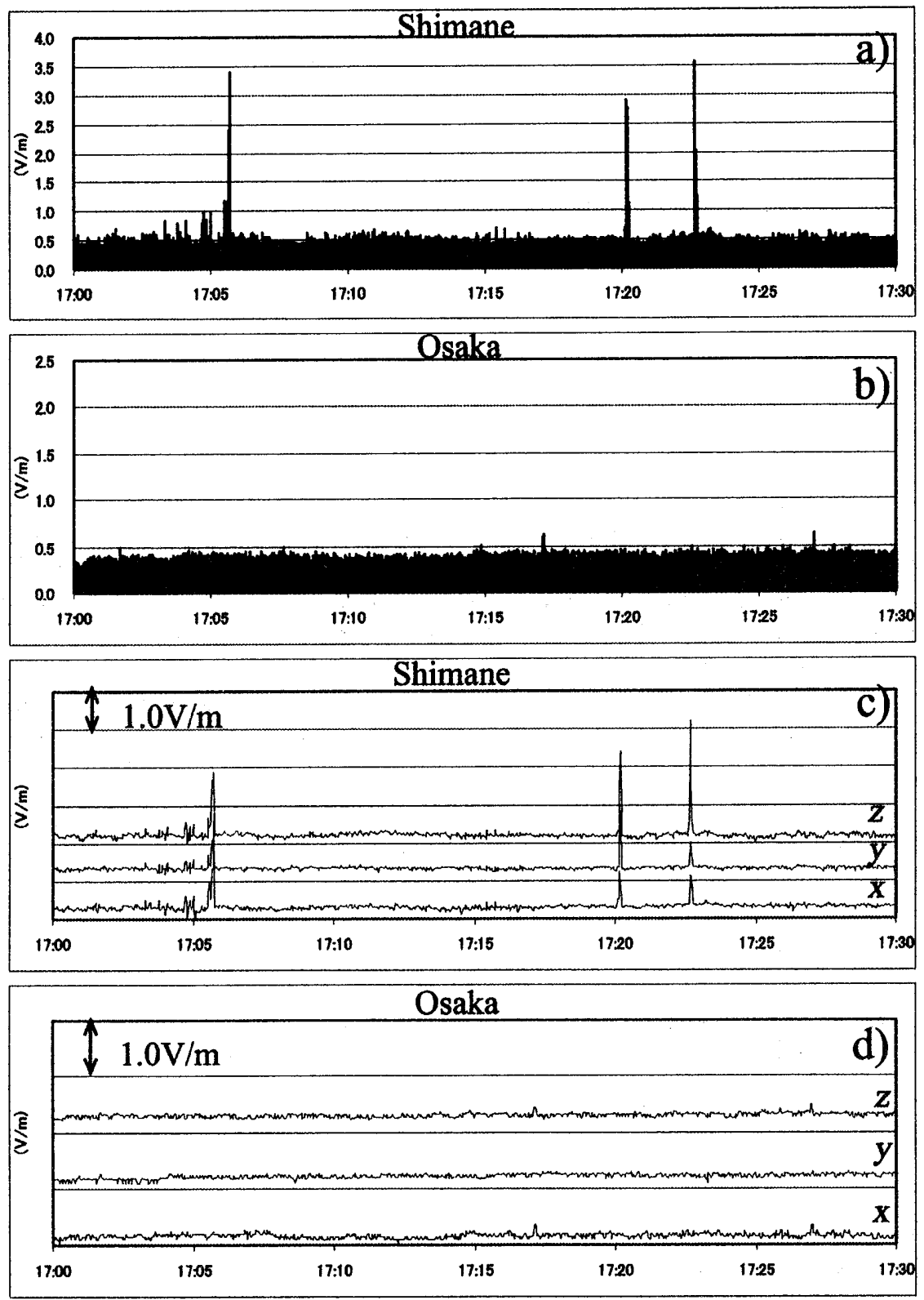

Fig.8: Results of high frequency observation at Shimane (a) and Osaka (b) on March 23, 2001, one day before of the Geiyo Earthquake (Mar. 24, 2001 15:28 JST). Only the Shimane site detected unusually intense EM spikes. 3-D components of the electric fields are indicated in (c) and (d), respectively. 
probability of coincidence by chance would be $P_{1} \times P_{2}=3.22 \times 10^{-8}$. This corresponds almost once in a year. Therefore, it is not clear with this rough estimation, if both phenomena are related or not. Clearly simultaneous measurements at different sites are required for further research.

\subsection{Quantitative evaluation of EM waves at far field}

Observed pulses before two EQs might not be the preseismic activity. However, we here assume that they were potential precursor signals and emitted from epicenters to estimate the source power. We adopt a model of SEMS that charge generation due to rapid stress changes gives EM pulses (Ikeya, Takaki et al., 1997).

Let us assume a dipole emission around the epicenter, which would experience local stress changes before the main shock. Electric field $E$ away from the dipole in the wave zone is written by,

$$
E=\frac{\sqrt{45 P_{\mathrm{d}}}}{R} \sin \theta
$$

where $P_{\mathrm{d}}$ is the emitting power assuming a single dipole source, $R$ is the distance from the emitting area to the detection point and $\theta$ is the radiation angle. Maximum dipole emission is obtained at $\theta=90^{\circ}$. Note that actual EM emissions would be the summations of multi-dipole emissions distributed in the stress changing zones with the total volume of $L^{3}$. Consequently, observed EM waves are composed of pulse trains originating from various dipoles. While, the EM sensors detect the summation of EM pulses with $400 \mathrm{~ms}$ intervals. Considering the effective duration of pulse trains, $t$, the energy density of an EM waves per a unit time and a unit source volume, $W_{\mathrm{d}}$, will be written

$$
W_{\mathrm{d}}=\frac{P_{\mathrm{d}}}{L^{3}} \cdot t
$$

The elastic energy density in rock is obtained by the summation of energy due to compression and shear strains

$$
W_{\mathrm{e}}=\frac{1}{2} \times K \times\left(\frac{\Delta V}{V}\right)^{2}+\mu \sum e^{2}
$$

where $K$ is bulk modulus, $\Delta V / V$ is the fractional change in volume, $\mu$ is shear modulus and $e$ is shear strain. Usually, fracture of a rock occurs at approximately $\Delta V / V=10^{-4}$ or $e=10^{-4}$. Assuming typical values, $K=5 \times 10^{10} \mathrm{~Pa}$ and $\mu=3 \times 10^{10} \mathrm{~Pa}$, we obtain the energy densities due to compression and shear strain to be 250 and $300 \mathrm{~J} / \mathrm{m}^{3}$, respectively. Total elastic energy in the volume will be given by,

$$
U=W_{\mathrm{e}} \cdot L^{3}
$$

We assume the displacement is negligible in preseismic stress change, and tectonic stress was maintained through the fault zone, because local fractures related to preseismic activity are hardly recorded. Such local fractures must be of the types that are not detected by the current network of seismometers. If the fracture zone is small, the fracture is fast enough to cause only a high frequency of seismic waves that rapidly decay. Such waves would not be recorded with conventional seismometers.

We will sketch the density of electric power and elastic energy as a function of the volume of preseismic stress changing zones, $L^{3}$. Table 1 shows the cases of the Western Tottori EQ and the Geiyo EQ. The distance from epicenter to the measuring site was $190 \mathrm{~km}$ (epicenter to Osaka) and $150 \mathrm{~km}$ (epicenter to Shimane), respectively. In the case of the Geiyo EQ, the observed pulse field was $E=3.0 \mathrm{~V} / \mathrm{m}$ after subtracting the background of $0.5 \mathrm{~V} / \mathrm{m}$. Therefore, 
Table 1: Estimation of EM energy density. Observed EM fields before earthquakes are converted to the source energy densities assuming pulsewidth of $1 \mathrm{~s}$ and emissions from multi dipoles distributing in the volume of $L^{3}$.

\begin{tabular}{|cc|cc|cc|}
\hline SEMS FIELD & \multicolumn{2}{|c|}{$\begin{array}{l}\text { The Western Tottori EQ } \\
E_{\max }=0.92 \mathrm{~V} / \mathrm{m}, R=190 \mathrm{~km}\end{array}$} & \multicolumn{2}{|l|}{$\begin{array}{l}\text { The Geiyo EQ } \\
E_{\max }=3.0 \mathrm{~V} / \mathrm{m}, R=150 \mathrm{~km}\end{array}$} \\
\hline \multicolumn{2}{|c|}{ Assumed $L$} & \multicolumn{2}{|c|}{ Source EM energy density $P_{\mathrm{d}} t / L^{3}(t=1 \mathrm{~s}$ assumed $)$} \\
\hline $10^{1}$ & $(\mathrm{~m})$ & $6.79 \times 10^{5}$ & $\left(\mathrm{~J} / \mathrm{m}^{3}\right)$ & $4.5 \times 10^{6}$ & $\left(\mathrm{~J} / \mathrm{m}^{3}\right)$ \\
\hline $10^{2}$ & $(\mathrm{~m})$ & $6.79 \times 10^{2}$ & $\left(\mathrm{~J} / \mathrm{m}^{3}\right)$ & $4.5 \times 10^{3}$ & $\left(\mathrm{~J} / \mathrm{m}^{3}\right)$ \\
\hline $10^{3}$ & $(\mathrm{~m})$ & $6.79 \times 10^{-1}$ & $\left(\mathrm{~J} / \mathrm{m}^{3}\right)$ & 4.5 & $\left(\mathrm{~J} / \mathrm{m}^{3}\right)$ \\
\hline $10^{4}$ & $(\mathrm{~m})$ & $6.79 \times 10^{-4}$ & $\left(\mathrm{~J} / \mathrm{m}^{3}\right)$ & $4.5 \times 10^{-3}$ & $\left(\mathrm{~J} / \mathrm{m}^{3}\right)$ \\
\hline
\end{tabular}

we obtain $P_{\mathrm{d}}=4.5 \times 10^{9} \mathrm{~W}$. We take into account of the performance of EMR-20 and assume the pulse duration was of the order of one second. Then the emission density would be $4.5 \mathrm{~J} / \mathrm{m}^{3}$ assuming radiation volume of $V=1 \mathrm{~km}^{3}$. This corresponds to $1 \%$ of elastic energy density in the unit volume.

\subsection{Emission from one quartz particle}

If the SEMS is related to the preseismic stress changes, free charge $q(t)$ will appear with pulse-like forms for each stress changing event as described by (Ikeya and Takaki, 1996; Ikeya, Takaki et al., 1997),

$$
\frac{\mathrm{d} q}{\mathrm{~d} t}=-\alpha\left(\frac{\mathrm{d} \sigma}{\mathrm{d} t}\right)-\frac{q}{\varepsilon \rho}
$$

where $q$ is the released charge, $\alpha$ is the effective piezo-coefficient of quartz, $2.0 \times 10^{-12} \mathrm{C} / \mathrm{N}$ (Kondorashev, 1980; Sasaoka et al., 1998), $\sigma$ is the stress, $\varepsilon$ is the dielectric constant of the surrounding rock, and $\rho$ is the electric resistivity of the rock.

A set of unbound charges with different polarity is considered as a dipole at position $x$ and time $t$ as $p(x, t)=q(x, t) \cdot \mathrm{d} l$, where $\mathrm{d} l$ is the length of a dipole and assumed to be the length of a quartz particle. For simplicity, we focus the fast movement of charges, neglecting the second term of Eq. (5) and assume a sinusoidal stress change,

$$
\sigma(t)=\sigma_{0} \sin \omega t
$$

Charge is described as follows, assuming piezo-coefficient $\alpha$ is constant,

$$
q(t)=-\alpha \sigma(t)=-\alpha \sigma_{0} \sin \omega t
$$

The dipole moment formed by a single quartz particle is described by,

$$
p(t)=q(t) \cdot \mathrm{d} \boldsymbol{l}
$$

From Eqs. (7) and (8),

$$
\ddot{p}(t)=\ddot{q}(t) \cdot \mathrm{d} l=\alpha \omega^{2} \sigma_{0} \sin \omega t \cdot \mathrm{d} l
$$

The electric field is described using the formula of multipole expansion as the summations of static field $\left(E_{1}\right)$, induced field $\left(E_{2}\right)$ and far field $\left(E_{3}\right)$.

$$
E_{\text {total }}(x, t)=E_{1}(x)+E_{2}(x, t)+E_{3}(x, t)
$$


For far field at position $R$,

$$
\boldsymbol{E}_{3}(x, t)=\frac{1}{4 \pi \varepsilon_{0}}\left(-\frac{\ddot{p}\left(t-\frac{R}{c}\right)}{c^{2} R}+\frac{x\left(x \cdot \ddot{p}\left(t-\frac{R}{c}\right)\right)}{c^{2} R^{3}}\right)
$$

where $c$ is the speed of light. We also assume here that observed EM field $E=\left|E_{3}\right|$. Using a cylindrical coordinate, at the wave zone and $R<<$,

$$
\boldsymbol{E}_{\theta}=\frac{1}{4 \pi \varepsilon_{0}} \frac{\ddot{\boldsymbol{p}}}{c^{2} R} \sin \theta, \text { similarly, } \boldsymbol{H}_{\varphi}=\frac{1}{4 \pi} \frac{\ddot{\boldsymbol{p}}}{c R} \sin \theta
$$

The Poynting's vector to the $r$ direction at wave zone is

$$
\boldsymbol{S}_{\mathrm{r}}=\boldsymbol{E}_{\theta} \boldsymbol{H}_{\varphi}=\frac{\ddot{\boldsymbol{p}} \sin ^{2} \theta}{(4 \pi c R)^{2} c \varepsilon_{0}}
$$

Total emission from a single dipole is

$$
I=\int_{0}^{\pi} S_{\mathrm{r}} 2 \pi r^{2} \sin \theta \mathrm{d} \theta=\frac{1}{6 \pi \varepsilon_{0} c^{3}} \ddot{p}^{2}
$$

The stress change time $(2 \pi / \omega)$ is expressed by $\mathrm{d} l / V_{\mathrm{s}}=0.5 \mu$ s assuming $\mathrm{d} l=1 \mathrm{~mm}$ and a wave velocity of $S$ waves $\left(V_{\mathrm{S}}=2000 \mathrm{~m} / \mathrm{s}\right)$. Substituting $\sigma_{0} \sim 10^{7} \mathrm{~Pa}$ and $\alpha=2 \times 10^{-12} \mathrm{C} / \mathrm{N}$, we obtain the effective power from a single quartz particle, $I=1.1 \times 10^{-3} \mathrm{~W}$. Then, the Poynting's vector to $r$ direction (assume $\sin \theta=1$ ) is obtained from (12).

$$
\left|S_{\mathrm{r}}\right|=\frac{1.3 \times 10^{-4}}{R^{2}} \quad[\mathrm{~W}]
$$

If the content of quartz in volume $L^{3}$ is $10 \%$, The number of quartz particles $n$ would be

$$
n=(L / \mathrm{d} l)^{3} \times 0.1
$$

To obtain the source power at the Geiyo EQ of $P_{\mathrm{d}}=4.5 \times 10^{9} \mathrm{~W}$, the number of involved particles should be $P_{\mathrm{d}} / S_{\mathrm{r}}(R=1) \sim 3.5 \times 10^{13}$. This corresponds to the $L$ of $70 \mathrm{~m}$ assuming eq.(15) and $\mathrm{d} l=1 \mathrm{~mm}$. The duration of a single EM emission continues only $\mathrm{d} l / V_{\mathrm{S}}=0.5 \mu \mathrm{s}$, however the actual signal measured by EMR-20 continued at least 0.4 to 1 second. Therefore, the EM emissions due to stress changing phenomena considered here should occur repeatedly for about 1 second to explain the observed EM field. If the volume of $L^{3}$ deforms as continuum and quartz contained in this volume act coherently, enough EM field will be radiated. Unfortunately, it is not clear that such process can really occur, however, following speculation can be made.

Considering usual seismic stress drop of $10 \mathrm{MPa}$ and typical stress drops about $100 \mathrm{MPa}$ at fracture of rocks, seismic asperities that keep a fault until final slips will be one-tenth of all the area of the fault plane. The other nine-tenths regions can be considered as the potential stress changing zones, where local stresses may be exchanged intermittently before the final slip and EM pulses would be emitted repeatedly. In that case, coseismic EM emissions would be limited because most of the geo-stress is focused on the relatively small asperity area at the main faulting.

The above estimation is based on several assumptions including alignment of quartz, coherent stress changes, and no attenuation. Therefore, the mechanism of SEMS is not fully resolved yet and further studies are required. The hypothesis of ferroelectric phase shifts of piezo-elements to form long dipoles may also account for this problem (Ikeya, 1999). More 
detailed results considering attenuation factors, near field EM field, and charge relaxation process will be separately published.

The electric field should be monitored as a function of distance from the epicenter. If the EM field were not directly coming from the epicenter, we must consider other routes of EM waves such as ground waveguides (Ikeya, Kinoshita et al., 1997; Ikeya, Matsumoto et al., 2000).

\section{Summary}

A system measuring the environmental electromagnetic field at wide bandwidths has been developed and continuously operated at Osaka and Shimane. The system characteristics for detection of pulsed electromagnetic fields were examined and the devices were proved to have an almost flat response at pulse width longer than 0.4 seconds. The activity of catfish in response to environmental electromagnetic fields is successfully monitored using a bio-electromyogram, which enables us to record the activity quantitatively.

Unusual pulse fields and synchronous vigorous activity of catfish were observed at Osaka 8 days before the Western Tottori EQ. Also, intense EM pulses were observed at Shimane one day before the Geiyo EQ. It is important to have more sites to judge the relation between those phenomena and EQs. All neighboring sites should be examined using "coincidence analysis" to filter out local phenomena such as static discharge or artificial noises. Systematic, direction sensitive and simultaneous observations at different sites and at wide frequencies are required to obtain reliable scientific data.

It must be pointed out that the views on EQ precursors are biased by premature application for practical EQ prediction. Phenomenological and quantitative analysis of various EQ precur-

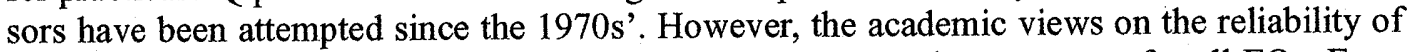
precursors to EQs became negative because such precursors do not appear for all EQs. Even when such anomalies appear, it is difficult to determine their reliability in real time and quite impossible to announce an EQ prediction considering the serious effects to the society in case of false prediction. However, particularly required in this field are continuous observation and physical hypotheses explaining such phenomena.

\section{References}

Asada, T. Baba, H., Kawazoe, M and Sugiura, M., An attempt to delineate very low frequency electromagnetic signals associated with earthquakes, Earth Planets Space, 53, 55-62, 2001. Asano, M., Catfish can sense electricity, Earthquake Journal, 26, 52-59,1998, In Japanese.

Fraser-Smith, A.C., A. Bernardi, P.R. McGill, M. E. Ladd, R. A. Helliwell, and O.G. Villard, Jr., Low-frequency magnetic field measurements near the epicenter of the $\mathrm{M}_{\mathrm{S}} 7.1$ LomaPrieta earthquake, Geophys. Res. Lett., 17. 1465-1468, 1990.

Hayakawa, M. Editor. Atmospheric and ionospheric electromagnetic phenomena associated with earthquakes, Terra Sci. Pub. Co., Tokyo, 996p, 1999.

Hayakawa, M. and Fujinawa, Y. Editors., Electromagnetic phenomena related to earthquake prediction, Terra Sci. Pub. Co., Tokyo, 677p, 1994.

Hayakawa, M., Molchanov, O. A., Ondoh, T. and Kawai, E., Precursory signature of the Kobe EQ on VLF subionospheric signal, J. Atom. Electr. 16, 247-257, 1996.

Matsumoto, H. Ikeya, M. and Yamanaka, C., Analysis of barber-pole color and speckle noises recorded 6 and half hours before the Kobe Earthquake, Jpn. J. Appl. Phys. 37, L1409$1411,1998$. 
Ikeya, M., Earthquake precursors due to seismic electromagnetic signals (SEMS), Recent Res, Devel. Applied Phys., 2, 109-127, 1999.

Ikeya, M., Furuta, H., Anzai, H. and Kajiwara N., Electric field effects on rats and sparrows for seismic animal anomalies (SAABs), Jpn. J. Appl. Phys., 65, 4587-4594, 1996.

Ikeya, M., Kinoshita, Y., H. Matsumoto, Takaki S. and Yamanaka C., A model of experiment of EM wave propagation over long distance using waveguide terminology, Jpn. J. Appl. Phys., 37, L1558-L1561, 1997.

Ikeya, M., Matsuda, T. and Yamanaka, C., Reproduction of mimosa and clock anomalies before earthquakes: "Are they Alice in Wonderland Syndrome?" , Proc. Japan Acad., 74., Ser.B, 60-64, 1998.

Ikeya, M. and Matsumoto, H., Reproduced earthquake precursor legends using a Van de Graaff electrostatic generator, candle flame and dropped nails, Naturwissenschaften, 84, 539-541, 1997.

Ikeya, M. and Matsumoto, H., Duplicated earthquake precursor anomalies of electric appliances, South China J. Seismology, 18, 53-57, 1998.

Ikeya, M., Matsumoto, H., Huang, Q. and Takaki. S., Theoretical Scaling Laws for Fault length, Seismic Electromagnetic Signals (SEMS) and Maximum Appearance Area, J. of Earthquake Prediction Research, 8, 351-360, 2000.

Ikeya, M. and Takaki, S., Electromagnetic model of a fault for earthquake lightning, Jpn. J. Appl. Phys., 35, L355 - 357, 1996.

Ikeya, M., Takaki, S., Matsumoto, H., Tani A. and Komatsu T., Pulsed charge model of fault behavior producing seismic electric signals (SES), J. Circuit Systems, and Computers, 7, 153-164, 1997.

Ikeya, M., Takaki, S. and Takashimizu, D., Electric shocks resulting in seismic anomalous behaviors, J. Phys. Soc. Japan, 65, 710-712, 1996.

Kondrashev, S. N., The piezoelectric method of exploration, Nedra, Moscow, 1980, English translation by Volkoff, G.M., Univ. British Columbia, Vancouver, 202, 1991.

Kushida, Y. and Kushida, R., On a possibility of earthquake forecast by radio observations in the VHF band, RIKEN Review, 19, 152-160, 1998.

MacGorman, D. R. and Rust W.D., The electrical nature of storms, Oxford Univ. Press, Oxford, Ch. 5-12, 1998.

Sasaoka, H., Yamanaka, C. and Ikeya, M., Measurements of electric potential variation by piezoelectricity of granite, Geophys. Res. Lett. 25, 2225-2228, 1998.

Tributsch, H., Wenndie Schlangen Erwachen, Deutsche Verlags-Anstalt GmbH, Stuttgart, Ch. $8,1978$.

Wyss, M. and Booth, D. C. The IASPEI procedure for the evaluation of earthquake precursors, Geophys. J. Int., 131, 423-424, 1997.

(Received November 16, 2001; revised May 16, 2002; accepted May 23, 2002) 